\title{
ÉTUDE DE LA SUSCEPTIBILITÉ MAGNÉTIQUE DE L'OXYDE DE MANGANĖSE ET DU SULFURE DE MANGANĖSE EN POUDRE DANS L'ÉTAT ORDONNÉ
}

\author{
D. BLOCH et R. GEORGES \\ Laboratoire d'Electrostatique et de Physique du Métal, Grenoble, France \\ I. S. JACOBS \\ General Electric Research and Development Center, Schenectady, N. Y., U. S. A.
}

(Reçu le 5 février 1970)

\begin{abstract}
Résumé. - On a étudié expérimentalement la variation thermique de la susceptibilité magnétique en champ fort jusqu'à $70 \mathrm{kOe}$ de l'oxyde et du sulfure de manganèse. Dans le domaine magnétiquement ordonné, la direction d'antiferromagnétisme est modifiée sous l'action du champ magnétique appliqué ce qui conduit à une augmentation de la susceptibilité. On a observé des phénomènes de traînage de la susceptibilité. On discute des résultats expérimentaux en tenant compte des déplacements des parois des domaines et de la magnétostriction spontanée.
\end{abstract}

\begin{abstract}
An experimental study of the thermal variation of the high field magnetic susceptibility of manganese oxide and sulfide in fields up to $70 \mathrm{kOe}$ is reported. In the magnetically ordered region, the direction of antiferromagnetism is modified by the applied field, which leads to an increase of the magnetic susceptibility. Magnetic viscosity effects were also observed. A discussion of the experimental results is given which takes into account domain wall displacement and spontaneous magnetostriction.
\end{abstract}

Introduction. - A la température ambiante, l'oxyde de manganèse et le sulfure de manganèse présentent la structure cubique à faces centrées du chlorure de sodium. Dans ces composés, les orbitales $3 d$ des ions $\mathrm{Mn}^{++}$contiennent cinq électrons. Leur moment magnétique de spin est de $5 \mu_{B}$ et le moment orbital est nul. Les mesures magnétiques ont montré que $\mathrm{MnO}$ et $\mathrm{MnS}$ en poudre présentent un comportement antiferromagnétique avec des températures de Néel [1-2] voisines respectivement de $120^{\circ} \mathrm{K}$ et $150 \circ \mathrm{K}$. On admet généralement que les interactions d'échange entre premiers et entre seconds voisins suffisent à décrire les propriétés de ces composés [3-4]. En 1951, Shull et al. ont mis en évidence, par diffraction des neutrons [5], la nature de l'arrangement antiferromagnétique dans $\mathrm{MnO}$. Celui-ci est constitué de plans ferromagnétiques (111), le sens des moments étant inversé entre deux plans consécutifs (arrangement antiferromagnétique de deuxième espèce). Un cristal se divise donc en domaines antiferromagnétiques, dits domaines $T$, en raison de la multiplicité de la direction des plans (111). Kaplan a partiellement résolu le problème de la direction d'antiferromagnétisme [6] que nous appellerons $\Delta$ dans la suite, en montrant que l'énergie d'interaction dipolaire est minimale lorsque $\Delta$ est parallèle aux plans ferromagnétiques; une étude effectuée ultérieurement par Roth [7], par la diffraction des neutrons, a confirmé ce résultat. Cependant, au-dessous de la température de Néel, la susceptibilité magnétique de $\mathrm{MnO}$ en poudre est une fonction croissante du champ appliqué [8]. Ce fait suggère l'existence d'un processus de rotation de la direction d'antiferromagnétisme [9] (dit encore «spin flotting ») sous l'action du champ appliqué. Mais, le terme d'anisotropie dipolaire ne suffit pas à expliquer ce comportement puisqu'il existe toujours une direction d'antiferromagnétisme, parallèle aux plans (111) et perpendiculaire au champ appliqué.

D’autre part des études récentes ont mis en évidence le rôle joué par les effets de magnétostriction spontanée dans $\mathrm{MnO}$ et dans $\mathrm{MnS}[10,11,12]$. Les modifications des paramètres de la maille, et la variation thermique des interactions d'échange ont une incidence sur les propriétés magnétiques, et en particulier sur les susceptibilités.

Pour répondre à l'ensemble des questions ainsi soulevées, nous avons entrepris l'étude de la susceptibilité magnétique de l'oxyde de manganèse et du sulfure de manganèse dans le domaine ordonné. Nous avons utilisé pour cette étude le champ magnétique produit par une bobine sans fer, du type 
Wood, refroidie à l'eau [13]. Les mesures ont été effectuées par la méthode d'extraction axiale. Les résultats sont corrigés de la susceptibilité diamagnétique des ions $\mathrm{Mn}^{++}, \mathrm{O}^{--}$et $\mathrm{S}^{--}$, soit $-1,6 \times 10^{-6}$ u.e. m./g. pour $\mathrm{MnO}$ et $-0,6 \times 10^{-6}$ u.e.m./g pour $\mathrm{MnS}$ [14]. La précision des expériences est estimée à $\pm 0,6 \times 10^{-6}$ u. e. m./g.

Etude expérimentale de la susceptibilité magnétique de MnO en poudre. - Cette étude a été réalisée avec une poudre de même origine que celle utilisée par Roth pour l'étude de $\mathrm{MnO}$ par diffraction des neutrons [7]. Elle est dépourvue d'impuretés ferromagnétiques. La grosseur des grains est telle que l'on peut supposer, par analogie avec $\mathrm{NiO}[15,16,17,18]$ que chacun d'eux est constitué d'un seul domaine $T$ et que les phénomènes caractéristiques des poudres en grains fins ne peuvent pas prendre place. Les mesures à température constante sont effectuées à partir d'un état de référence présentant en champ nul une distribution parfaitement aléatoire des plans ferromagnétiques (111) et des directions d'antiferromagnétisme [19].

Les résultats obtenus lors des mesures de la susceptibilité de $\mathrm{MnO}$ en poudre en champs magnétiques croissants, à partir de l'état de référence ont déjà fait l'objet d'une note [20] (Fig. 1). Dans le domaine paramagnétique, l'accord avec les mesures antérieures est satisfaisant [18]. Au-dessous de la température de Néel, la susceptibilité antiferromagnétique $\chi_{\mathrm{p}}$ de la poudre croît avec le champ appliqué jusqu'à 50000 Oe environ. Pour les valeurs supérieures du champ, elle reste constante. Nous admettons que

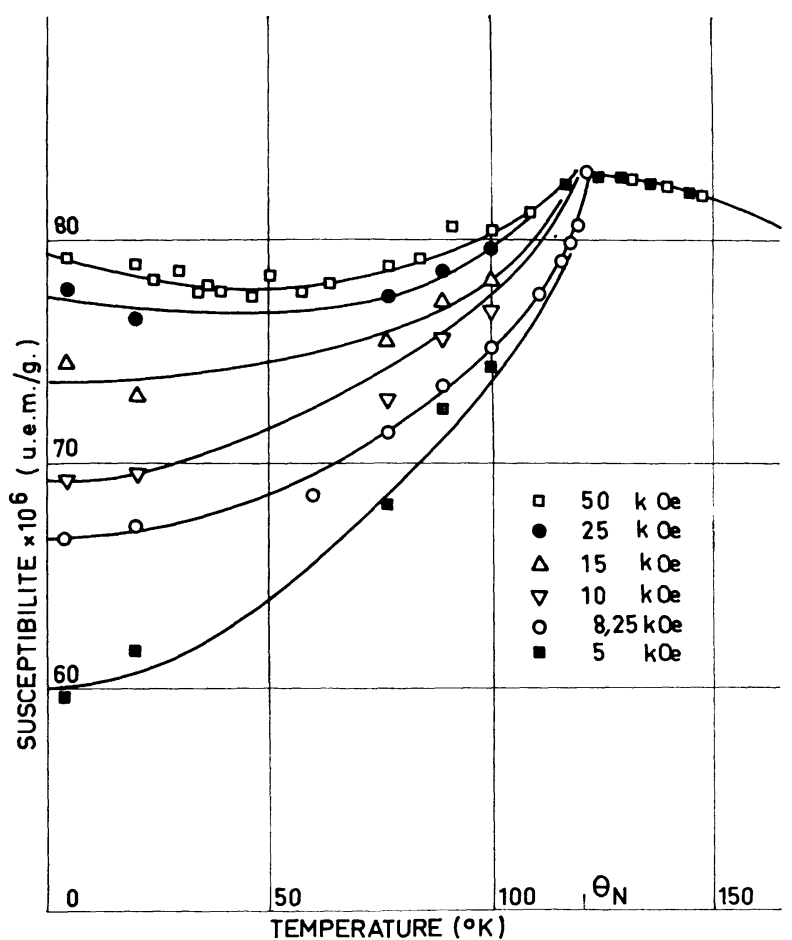

FIG. 1. - Susceptibilité magnétique de $\mathrm{MnO}$ en poudre (référence 20). dans ces conditions, à l'intérieur de chaque grain de la poudre, la direction d'antiferromagnétisme $\Delta$ est perpendiculaire au champ appliqué, et que la susceptibilité magnétique mesurée est égale à la susceptibilité perpendiculaire, $\chi_{\perp}$. Nous avons ainsi à $4,2^{\circ} \mathrm{K}$ :

$$
\chi_{\perp}=(79,0 \pm 0,6) \times 10^{-6} \text { u. e. } \mathrm{m} . / \mathrm{g}
$$

avec une meilleure précision que lors des évaluations antérieures [21-22]. Lorsque la température croît, $\chi_{\perp}$ diminue légèrement. Sa variation thermique présente un minimum très étalé autour de $55{ }^{\circ} \mathrm{K}$. La valeur de $\chi_{\perp}$ est alors de $78 \times 10^{-6}$ u. e. m./g; cette valeur croît ensuite jusqu'à la température de transition où elle est de $83 \times 10^{-6}$ u. e. m./g.

Dans un autre ensemble de mesures, nous avons constaté que les susceptibilités magnétiques, mesurées en faisant décroître le champ à partir de 50000 Oe, sont supérieures aux susceptibilités mesurées en champs croissants à partir de l'état de référence (Fig. 2). Si l'on cesse de faire décroître le champ en le maintenant constant, par exemple à 6000 Oe, la susceptibilité magnétique diminue peu à peu et tend vers la valeur mesurée en champs croissants avec une constante de temps de 40 minutes environ. L'appareillage utilisé ne permet que des variations très lentes du champ magnétique, de sorte qu'il est impossible de déduire des renseignements quantitatifs des courbes obtenues en champs décroissants.

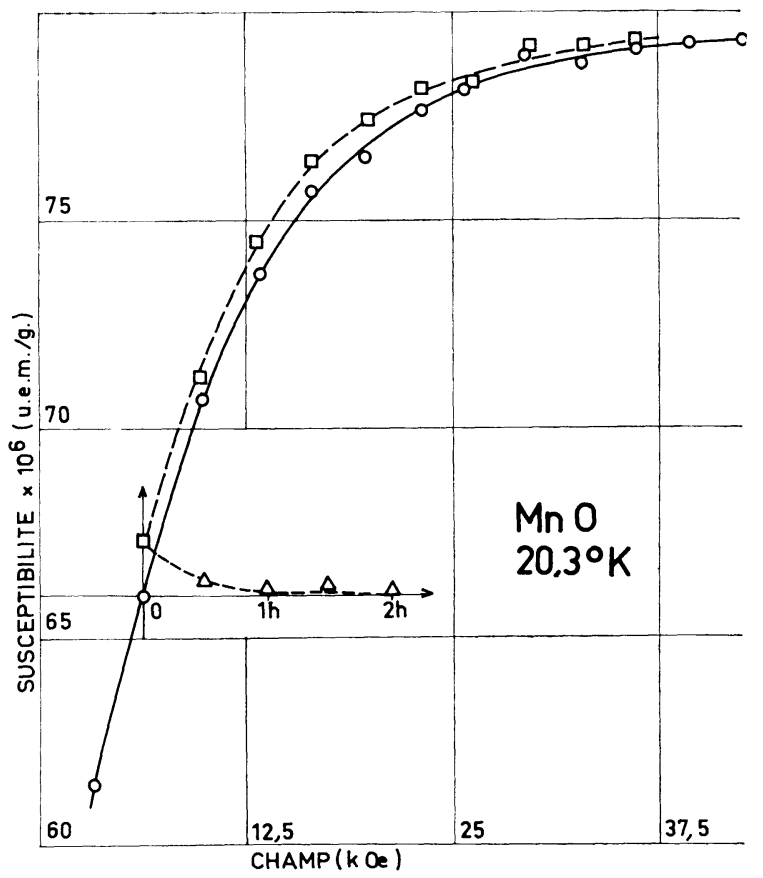

FIG. 2. - Traînage magnétique en fonction du champ dans MnO.

Enfin, nous avons étudié sous champ constant la variation thermique de la susceptibilité $\chi_{\mathrm{p}}$ en faisant croître, puis décroître la température. En franchissant le point de transition $\left(120^{\circ} \mathrm{K}\right)$ dans le sens des températures décroissantes, les valeurs de $\chi_{p}$ 
sont supérieures à celles que l'on obtient à températures croissantes (Fig. 3). Si après avoir refroidi rapidement l'échantillon au-dessous de la transition on maintient sa température à une valeur fixe, on observe que la susceptibilité décroît peu à peu et tend vers la valeur mesurée à températures croissantes (la même expérience effectuée sur le pyrophosphate de manganèse n'a rien révélé d'analogue, de sorte que les effets observés ne semblent pas liés à la présence d'un gradient de température ou à un retard de la mise en équilibre thermique). Il semble que l'on doive rapprocher cet effet de l'observation rapportée par Rodbell, et Owen [23] : lorsqu'on refroidit rapidement une poudre d'oxyde de manganèse au-dessous de sa température de transition, le dédoublement de certaines raies du diagrammes de DebyeScherrer, qui caractérise l'apparition de la distorsion rhomboédrique liée à l'ordre antiferromagnétique, n'apparaît qu'au-delà de 40 minutes environ. Nous verrons plus loin que ces deux phénomènes peuvent trouver une explication commune.
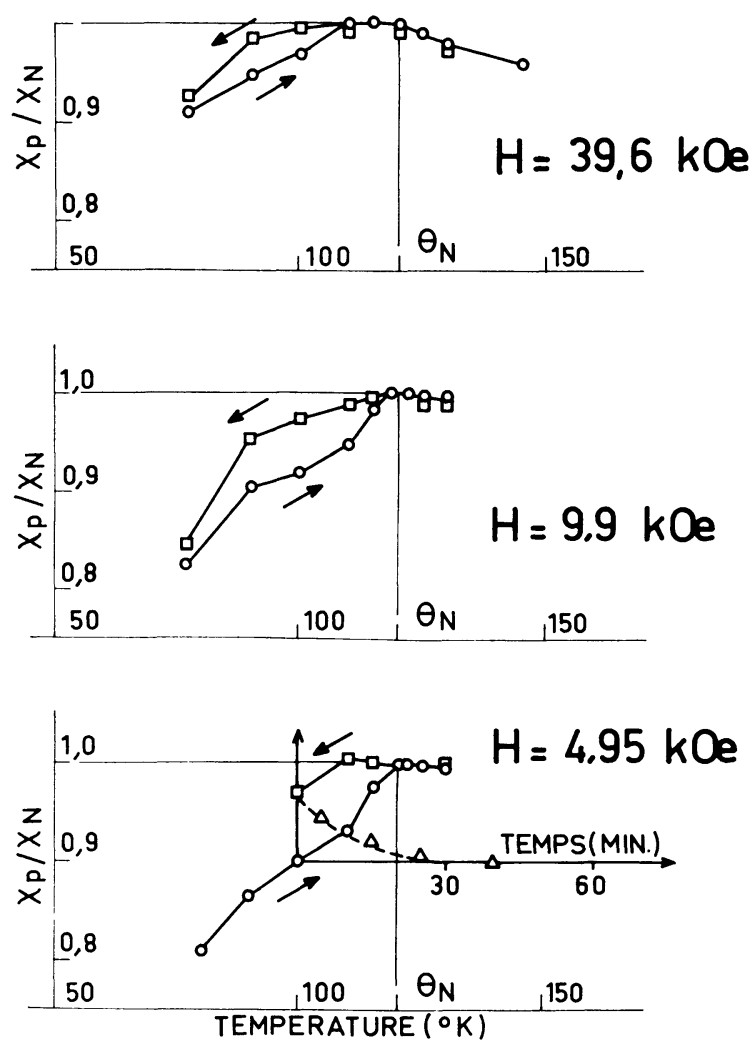

FIG. 3. - Traînage magnétique en fonction de la température dans $\mathrm{MnO}$.

Interprétation. - Dans ce paragraphe, nous donnons une interprétation de la variation thermique de la susceptibilité perpendiculaire $\chi_{\perp}$ de $\mathrm{MnO}$, et nous confrontons avec nos résultats expérimentaux le modèle proposé par Keffer et O'Sullivan pour analyser les phénomènes de «spin-flopping», Enfin, nous proposons diverses interprétations des phénomènes de traînage et de retard que nous avons observés.
Il est possible d'interpréter la variation thermique de la susceptibilité perpendiculaire en utilisant le modèle des ondes de spins aux basses températures et le modèle du champ moléculaire au-delà de $40^{\circ} \mathrm{K}$.

Lorsqu'on applique suivant la direction $O y$ (perpendiculaire à la direction $O z$ de facile aimantation) un champ magnétique $H$, faible devant le champ d'échange $H_{\mathrm{E}}$, mais fort devant le champ critique de «sping flopping " [22], les aimantations moyennes $\bar{\sigma}_{\mathrm{A}}$ et $\overline{\boldsymbol{\sigma}}_{\mathrm{B}}$ des sous-réseaux forment un angle $\Phi$, petit, avec la direction $\mathrm{Oz}$ (Fig. 4). Aux basses températures,

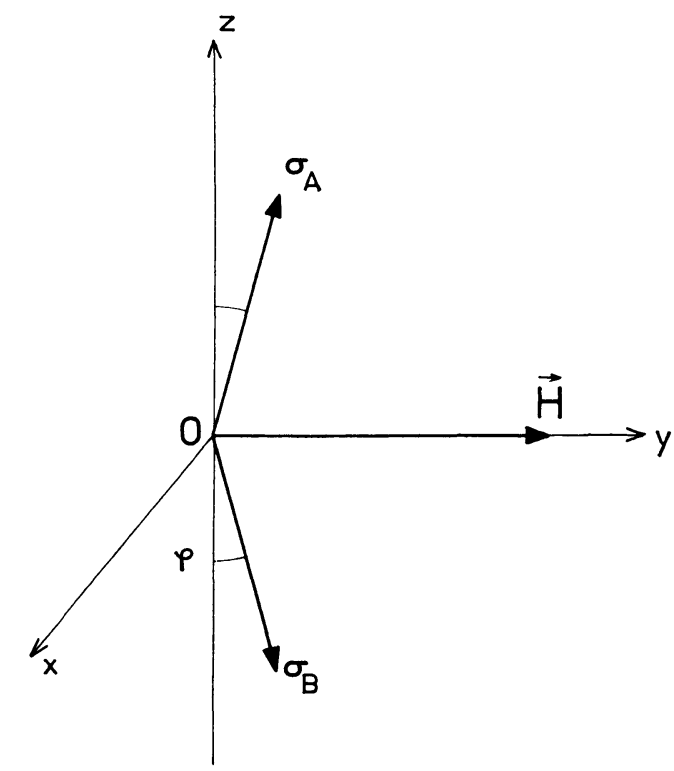

Fig. 4. - Effet d'un champ magnétique appliqué suivant une direction normale à la direction d'antiferromagnétisme.

la valeur de l'angle $\Phi$ rend minimale la partie de l'énergie totale qui dépend de cet angle :

$$
W(\Phi)=-H\left(\bar{\sigma}_{\mathrm{A}}^{z}-\bar{\sigma}_{\mathrm{B}}^{z}\right) \sin \Phi-W_{\mathrm{e}}(\Phi)
$$

où $\bar{\sigma}_{\mathrm{A}}^{z}$ et $\bar{\sigma}_{\mathrm{B}}^{z}$ sont les valeurs moyennes des projections suivant $O z$ des aimantations $\sigma_{A}$ et $\sigma_{B}$. Le deuxième terme de cette expression représente la variation avec $\Phi$ de l'énergie d'échange des paires d'ions antiparallèles. Soient deux ions antiparallèles $l$ et $m$, couplés par l'interaction d'échange $J$. En l'absence de champ extérieur, l'énergie moyenne de la paire $(l, m)$ est donnée par :

$$
J \overline{S_{l} \cdot S_{m}}=J\left(\overline{S_{l}^{x} S_{m}^{x}}+\overline{S_{l}^{y} S_{m}^{y}}+\overline{S_{l}^{z} S_{m}^{z}}\right) .
$$

La valeur de l'énergie moyenne de cette paire lorsqu'on applique le champ $H$ est égale à :

$$
\left.J \overline{S_{l \bullet} S_{m}}=J\left(\overline{S_{l}^{x} S_{m}^{x}}+\overline{\left(S_{l}^{y} S_{m}^{y}\right.}+\overline{S_{l}^{z} S_{m}^{z}}\right) \cos 2 \Phi\right)
$$

où les valeurs moyennes sont celles que l'on observe en champ nul. Nous avons négligé les quantités analogues à $\overline{S_{l}^{z} S_{m}^{y}}$. Cette approximation est moins rudimentaire que celle que l'on effectue dans le 
modèle du champ moléculaire et qui consiste à négliger les corrélations $\overline{S_{l}^{x} S_{m}^{x}}$ et $\overline{S_{l}^{y} S_{m}^{y}}$. Nous avons ainsi:

$$
\begin{aligned}
W(\Phi)= & -H N g \mu_{\mathrm{B}} S_{1}^{z} \sin \Phi \\
& \left.+6 N J_{1 \uparrow \downarrow}\left(\overline{S_{a}^{x} S_{b}^{x}}+\overline{\left(S_{a}^{y} S_{y}^{y}\right.}+\overline{S_{a}^{z} S_{b}^{z}}\right) \cos \Phi\right)_{1 \uparrow \downarrow} \\
& \left.\left.+6 N J_{2} \overline{\left(S_{a}^{x} S_{b}^{x}\right.}+\overline{\left(S_{a}^{y} S_{b}^{y}\right.}+\overline{S_{a}^{z} S_{b}^{z}}\right) \cos 2 \Phi\right)_{2} .
\end{aligned}
$$

L'indice $1_{\uparrow \downarrow}$ désigne une paire de premiers voisins $(a, b)$ antiparallèles et l'indice 2 une paire de seconds voisins.

En supposant que $\overline{S_{a}^{x} S_{b}^{x}}$ est égale à $\overline{S_{a}^{y} S_{b}^{y}}$ ce qui revient à négliger l'anisotropie, nous obtenons, au second ordre près en $\left(g \mu_{\mathrm{B}} H / J_{1 \uparrow \downarrow}\right)$

$$
\chi_{\perp}=\frac{N\left(g \mu_{\mathrm{B}}\right)^{2}\left(\overline{S_{1}^{z}}\right)^{2}}{12\left[J_{1 \uparrow \downarrow}\left(\overline{S_{a} S_{b}}+\overline{S_{a}^{z} S_{b}^{z}}\right)_{1 \uparrow \downarrow}+J_{2}\left(\overline{S_{a} S_{b}}+\overline{S_{a}^{z} S_{b}^{z}}\right)_{2}\right]} .
$$

Nous avons déterminé les variations thermiques

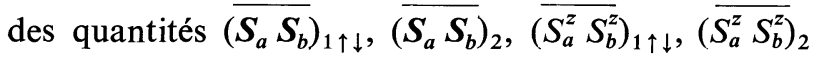
et $\overline{S_{1}^{z}}$ par un calcul d'ondes de spins non renormalisées [24] en adoptant les valeurs des interactions d'échanges déterminées par Lines et Jones [3]. On constate ainsi qu'entre $4,2^{\circ} \mathrm{K}$ et $40^{\circ} \mathrm{K}$, la susceptibilité $\chi_{\perp}$ décroît d'environ $1 \%$, ce qui est en accord avec l'expérience. Cette diminution s'explique par le fait que la création d'une onde de spin de faible ou de forte énergie diminue de la même quantité la composante $\overline{S_{1}^{z}}[25]$; par contre, les corrélations qui apparaissent au dénominateur de l'expression [6] sont moins affectées par les ondes de faible énergie qui sont les seules excitées aux basses températures ; ainsi le rapport qui exprime $\chi_{\perp}(T)$ commence par décroître lorsqu'on élève la température à partir du zéro absolu.

$\mathrm{Au}$ voisinage de la température d'ordre, le coefficient de champ moléculaire qui couple entre eux les sous-réseaux antiparallèles varie en fonction de la température par l'intermédiaire des variations thermiques de la distorsion $\alpha$ et du paramètre $a$ [10-11] (Fig. 5). Il en est de même, par conséquent, de la susceptibilité perpendiculaire, et nous obtenons:

$$
\frac{\chi_{\perp}(T)}{\chi_{\mathrm{p}}\left(\theta_{\mathrm{N}}\right)}=\frac{J_{1 \uparrow \downarrow}\left(\theta_{\mathrm{N}}\right)+J_{2}\left(\theta_{\mathrm{N}}\right)}{J_{1 \uparrow \downarrow}(T)+J_{2}(T)}
$$

Nous avons calculé la variation thermique de ce rapport en utilisant les résultats de mesures antérieures de la variation thermique de $a$ et de $\alpha$ ansi que de la variation des interactions d'échange en fonction des déformations de la structure cristallographique [10, 11, 12] (Fig. 5). Nous avons comparé les résultats ainsi obtenus et la variation thermique déterminée expérimentalement (Fig. 6). L'accord entre ces deux déterminations complètement indépendantes permet de souligner l'importance des effets de magnétostriction spontanée dans $\mathrm{MnO}$. Il faut noter aussi que
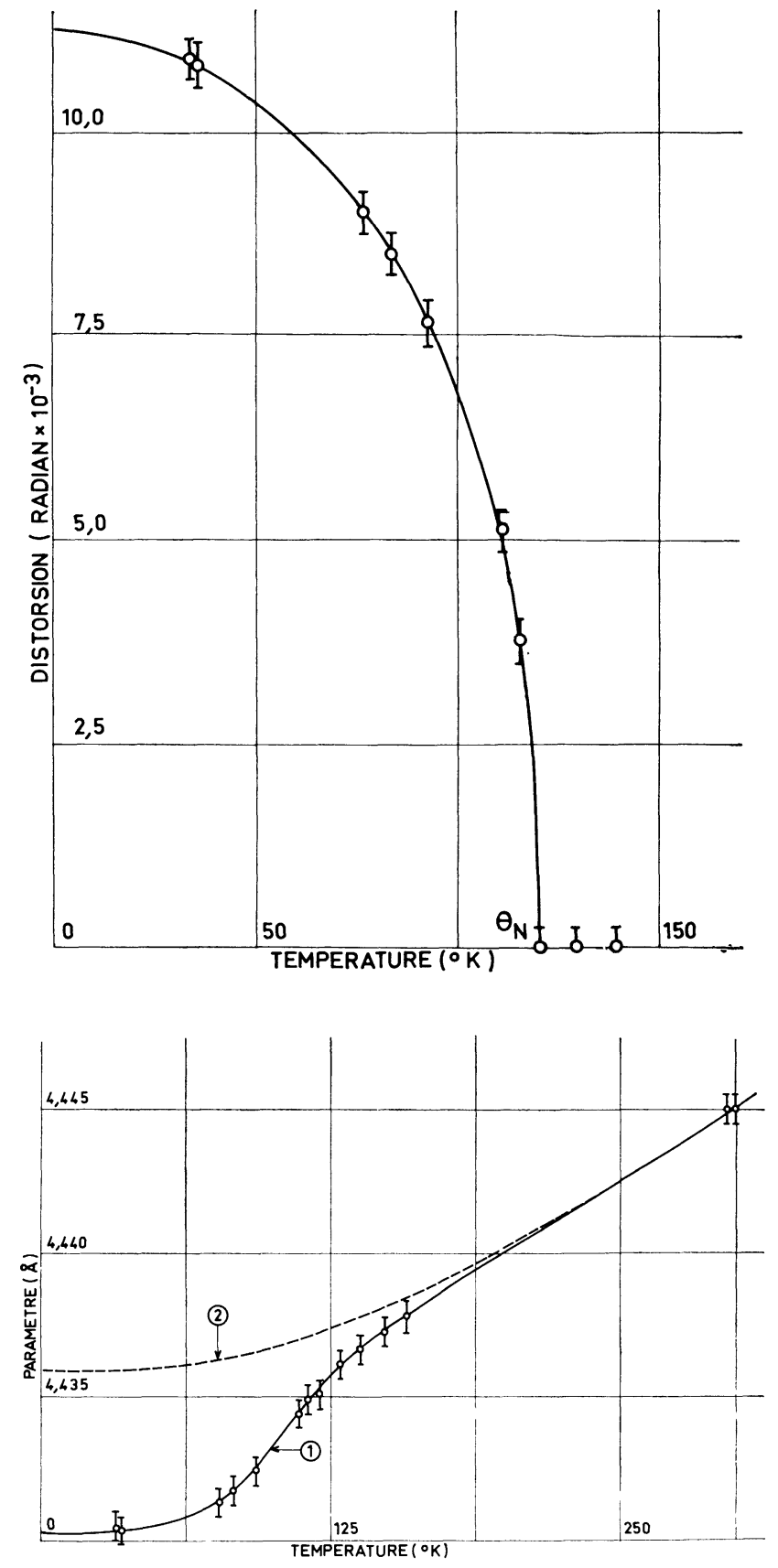

FIG. 5. - a) Variation thermique de la distorsion rhomboédrique $\alpha$ dans $\mathrm{MnO}$ (référence 11). b) Variation thermique du paramètre cubique $a$ dans $\mathrm{MnO}$ (référence 11) : 1. courbe expérimentale, 2. courbe calculée en l'absence de magnétostriction d'échange.

l'essentiel de la variation thermique de $\chi_{\perp}$ dans ce domaine de température est lié à l'apparition de la distorsion rhomboédrique.

Si l'on veut attribuer à un processus de spin flopping la variation de $\chi_{p}$ avec le champ appliqué, on est conduit à faire l'hypothèse qu'il existe au moins une direction de facile aimantation à l'intérieur des plans ferromagnétiques (111). On définit ainsi un nouveau type de domaines antiferromagnétiques (domaines $S$ ) correspondant aux diverses directions $\Delta$ rencontrées dans un même domaine $T$. Keffer et 


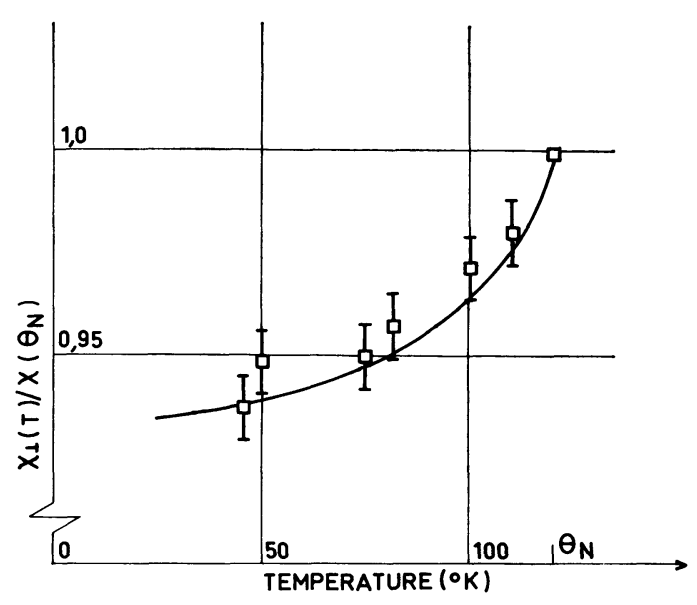

FIG. 6. - Variation thermique de la susceptibilité perpendiculaire de $\mathrm{MnO}$ au voisinage de la température de Néel.

O'Sullivan ont calculé la variation de la susceptibilité magnétique d'une poudre antiferromagnétique en fonction du champ magnétique appliqué [22]. Leur calcul suppose que les directions $\Delta$ sont initialement orientées de façon parfaitement aléatoire et que dans chaque domaine $S$ les spins tournent d'un même mouvement sous l'action du champ appliqué. On définit une susceptibilité réduite $\chi$, donnée par

$$
\chi=\frac{\chi_{\mathrm{p}}-\chi_{\|}}{\chi_{\perp}-\chi_{\|}} .
$$

Elle apparaît comme une fonction de la seule variable $h$, mesure du champ en prenant comme unité le champ critique $H_{\mathrm{c}}$ :

$$
H_{\mathrm{c}}=\left[2 m K_{2} /\left(\chi_{\perp}-\chi_{\|}\right)\right]^{1 / 2} .
$$

Dans cette relation $m$ et $K_{2}$ sont respectivement la multiplicité de la direction de facile aimantation dans le plan (111) et la constante d'anisotropie correspondante. Nous avons calculé les valeurs de $\chi(h)$ pour les valeurs 1 et $3 \mathrm{du}$ paramètre $m$ (Fig. 7). Le calcul pour le cas $m=3$ diffère un peu de celui effectué par Keffer et O’Sullivan [22].

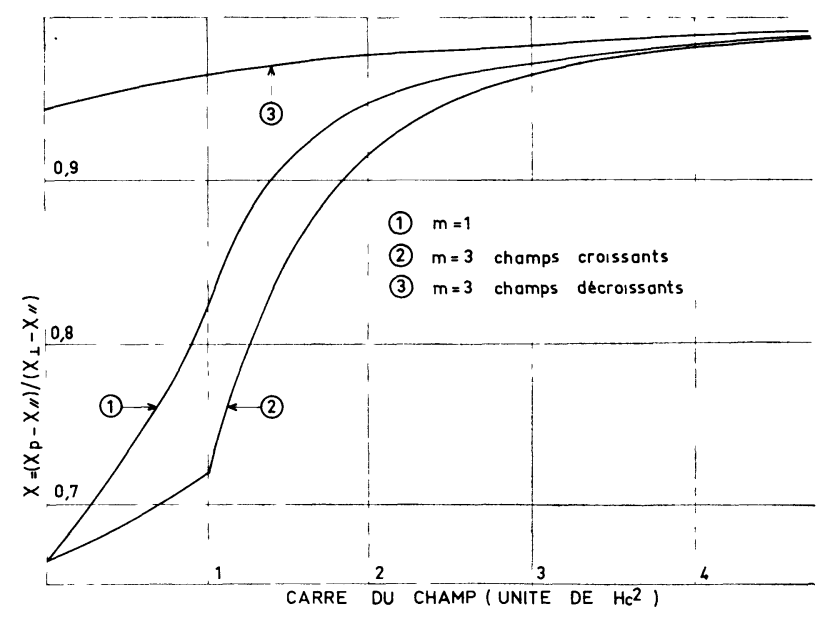

FIG. 7. - Variation théorique de $\chi$ en fonction de $h^{2}$.
S'il existe 3 directions de facile aimantation $(m=3)$, la courbe variation $\chi(h)$ présente un point anguleux pour la valeur $h=1$. En effet, lorsque le champ devient suffisamment intense, dans certains domaines $S$, la direction $\Delta$ bascule du voisinage d'une direction de facile aimantation au voisinage d'une autre, moins éloignée de la perpendiculaire au champ appliqué. De plus, si l'on supprime le champ après l'avoir porté à une valeur nettement supérieure à la valeur critique $(h \gg 1)$ les directions d'antiferromagnétisme dans chaque domaine $T$ se disposent suivant la direction de facile aimantation la plus proche de la normale au champ appliqué, et leur distribution n'est plus isotrope. La susceptibilité réduite $\chi$ est alors supérieure à la valeur $2 / 3$, correspondant à la répartition isotrope initiale. Si $m$ est égal à 1 , aucun de ces deux phénomènes ne se produit, et la variation correspondante de $\chi$ ne présente ni point anguleux ni hystérésis en fonction du champ.

Nous avons représenté (Fig. 8) l'isotherme théorique $\chi(h)$ pour $m=1$; nous avons représenté aussi les isothermes mesurées à $4,2^{\circ} \mathrm{K}$ et $77^{\circ} \mathrm{K}$, ajustées à la courbe théorique, par le choix de la valeur du champ critique, soit vers les champs forts, soit vers

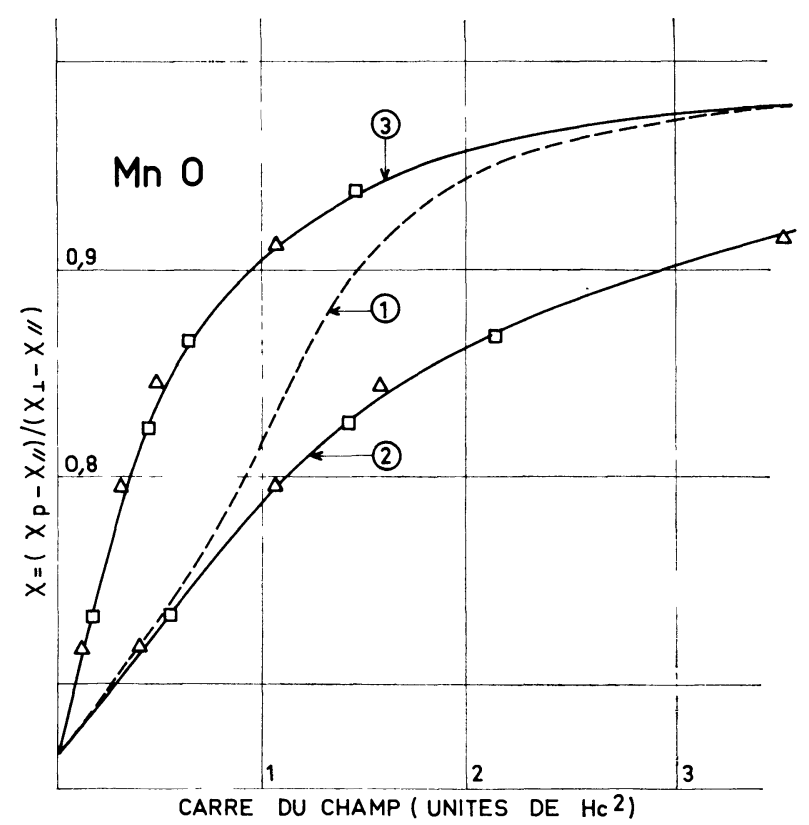

FIG. 8. - Variation expérimentale de $\chi$; comparaison avec la variation théorique pour $m=1: 1$. variation théorique, 2. ajustement aux champs faibles, 3. ajustement aux champs forts.

les champs faibles. On note que les variations expérimentales sont identiques en attribuant à $H_{\mathrm{c}}$ des valeurs dans le rapport.

$$
\frac{H_{\mathrm{c}}\left(77^{\circ} \mathrm{K}\right)}{H_{\mathrm{c}}\left(4,2^{\circ} \mathrm{K}\right)}=0,86 .
$$

Par contre, il est impossible d'amener en coïncidence les variations théoriques et expérimentales. 
Pour $m=3$, l'accord est encore moins satisfaisant, puisque, en particulier, l'expérience ne révèle pas l'existence de points anguleux.

Cette différence s'explique sans doute par le fait que le modèle de Keffer et O'Sullivan ne tient pas compte du déplacement des parois entre les domaines. Or, dans l'oxyde de nickel $\mathrm{NiO}$, et pour des champs magnétiques de l'ordre de 2000 Oe on peut observer de tels déplacements [26]. Ils peuvent rendre compte de la forte valeur de la susceptibilité parallèle à $4,2^{\circ} \mathrm{K}\left(10 \times 10^{-6}\right.$ u. e. $\left.\mathrm{m} . / \mathrm{g}\right)$ déterminée par extrapolation à partir de mesures effectuées dans des champs supérieurs à 2000 Oe.

L'analyse de la variation de la susceptibilité en fonction du champ appliqué, au moyen du modèle de Keffer et O'Sullivan, ne permet donc pas d'écarter l'une des hypothèses $m=1$ ou $m=3$, concernant la multiplicité des directions de facile aimantation dans le plan (111). L'étude du traînage magnétique fournit des éléments complémentaires pour discuter la valeur de $m$.

Dans l'hypothèse $m=3$ (3 directions équivalentes de facile aimantation) l'action d'un champ intense favorise le grossissement des domaines $S$ dans lesquels la direction d'antiferromagnétisme est proche de la normale au champ, au détriment des autres domaines $S$; elle provoque simultanément une rotation de toutes les directions d'antiferromagnétisme. Mais les domaines mal orientés peuvent subsister avec de très faibles volumes au voisinage de certains défauts, et reprendre peu à peu une plus grande extension après que l'on ait ramené le champ à une valeur plus faible. Cette hypothèse permet de rendre compte des phénomènes de traînage que nous avons observés; les diverses tentatives théoriques que nous avons faites pour évaluer l'anisotropie ternaire dans le plan (111) ont conduit à des ordres de grandeur nettement trop faibles.

L'hypothèse $m=1$ est incompatible avec la symétrie d'un réseau cristallin parfait, et ne peut favoriser le déplacement de parois entre les domaines $S$ (parois anti-phase). On peut cependant supposer que la présence de dislocations dans le réseau provoque des contraintes qui favorisent localement une des directions du plan (111). En présence d'un champ magnétique la rotation des moments entraîne le déplacement de certaines dislocations dont la présence s'opposerait à cette rotation. Lorsqu'on annule le champ, le mouvement de ces défauts vers leur répartition initiale pourrait provoquer le changement progressif des directions de facile aimantation et la variation correspondante de la susceptibilité $\chi_{\mathbf{p}}$.

On peut, d'autre part, proposer une explication commune au retard observé dans l'établissement de la distorsion rhomboédrique lorsqu'on abaisse la température de l'oxyde de manganèse au-dessous du point de transition, et à l'hystérésis de la susceptibilité en fonction de la température (Fig. 9) : dans chacun des grains, le franchissement de la tempéra-

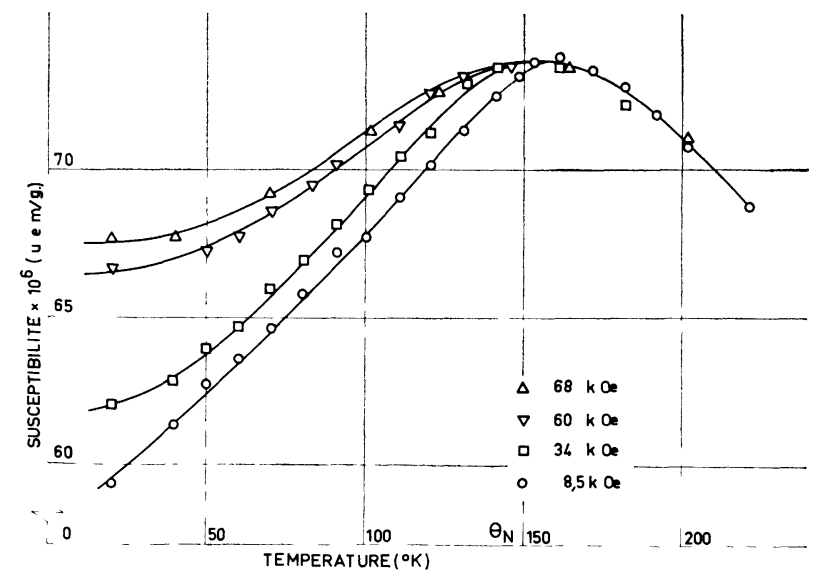

FIG. 9. - Susceptibilité magnétique de MnS en poudre.

ture de Néel provoque l'apparition de l'ordre antiferromagnétique. Initialement chaque grain peut compter plusieurs domaines $T$ et la distorsion rhomboédrique ne peut pas atteindre sa valeur normale (relation 5 de la référence 11) car des contraintes apparaissent alors dans les parois entre les domaines $T$. Le terme d'énergie correspondant diminue au fur et à mesure que la surface de ces parois diminue. De sorte que les grains tendent peu à peu à devenir monodomaines. Dans le même temps, la distorsion atteint la valeur normale et la susceptibilité $\chi_{\perp}$, liée à la valeur de la distorsion, comme nous l'avons déjà mis en évidence, tend vers la valeur mesurée en températures croissantes.

Etude des propriétés magnétiques du sulfure de manganèse. - La susceptibilité magnétique du sulfure de manganèse $\mathrm{MnS}$ a fait l'objet d'une étude analogue. Nous avons utilisé une poudre fournie par le Laboratoire de Chimie Minérale de la Faculté des Sciences de Lyon. La très faible aimantation rémanente, observée au-dessous de $45^{\circ} \mathrm{K}$ sur cet échantillon, peut être attribuée à la présence de l'oxyde ferrimagnétique $\mathrm{Mn}_{3} \mathrm{O}_{4}$ en proportion inférieure à $0,1 \%$. Les grains ont des dimensions de l'ordre du micron, mais ils ne présentent aucun des phénomènes caractéristiques du magnétisme des grains fins [27]. D'autre part, $\mathrm{MnS}$ ne semble pas présenter de distorsion rhomboédrique dans l'état ordonné [12], il est donc possible que chaque grain contienne plusieurs domaines $T$.

Nous avons reporté (Fig. 9) les résultats des mesures effectuées en champs croissants. Au-dessus de la température de Néel $\left(150^{\circ} \mathrm{K}\right)$, la susceptibilité $\chi_{\mathbf{p}}$ est indépendante du champ appliqué et les valeurs mesurées sont en accord avec les mesures antérieures effectuées dans le domaine paramagnétique [2]. Au-dessous de la température de Néel, la susceptibilité $\chi_{\mathbf{p}}$ augmente avec le champ magnétique. Nous n'avons pu atteindre la valeur à saturation : en effet, des mesures effectuées en champs pulsés jusqu'à $150 \mathrm{kOe}$ nous ont montré que cette valeur est atteinte pour des champs de l'ordre de $100 \mathrm{kOe}$. 
Nous pouvons attribuer au processus de «spin flopping » la variation de la susceptibilité magnétique avec le champ appliqué. Nous avons comparé les résultats expérimentaux aux variations prévues par le modèle de Keffer et O'Sullivan, en adoptant pour $\chi_{\perp}(T)$ la valeur de la susceptibilité mesurée à la température de Néel $\chi_{\mathrm{p}}\left(\theta_{\mathrm{N}}\right)$ : la faiblesse des effets de magnétostriction dans ce composé permet de supposer que $\chi_{\perp}$ varie peu en fonction de $T$. On constate que les isothermes $\chi(h)$ mesurées à $20,3{ }^{\circ} \mathrm{K}$ et $77 \mathrm{o} \mathrm{K}$ sont identiques à condition de choisir les champs critiques dans le rapport

$$
\frac{H_{\mathrm{c}}\left(77^{\circ} \mathrm{K}\right)}{H_{\mathrm{c}}\left(20,3^{\circ} \mathrm{K}\right)}=1,1 .
$$

Par contre, on observe comme dans le cas de l'oxyde de manganèse un désaccord entre l'expérience et le modèle de Keffer et O'Sullivan. La valeur de la susceptibilité parallèle à $4,2{ }^{\circ} \mathrm{K}$ calculée en extrapolant la variation de $\chi_{\mathrm{p}}$ vers les champs très faibles est voisine de $30 \times 10^{-6}$ u. e. m./g. Cette valeur très élevée semble confirmer l'existence des déplacements de parois entre les domaines $S$ pour les faibles valeurs du champ.
Conclusion. - Cette étude expérimentale n'avait pu être réalisée jusqu'ici faute d'obtenir des échantillons suffisamment purs ; elle a permis de montrer qu'il est impossible d'analyser les phénomènes associés au "spin flopping " sans tenir compte des mouvements de domaines. De sorte que des expériences sur des monocristaux rendus monodomaines $T$ par une contrainte externe, effectuées en champ statique et en champ pulsé, sont sans doute nécessaires pour apporter des renseignements décisifs sur la nature et l'importance de l'anisotropie dans le plan (111). En particulier, les valeurs du champ critique évaluées par comparaison avec le modèle de Keffer et O'Sullivan sont probablement largement trop faibles. Nous avons mis en évidence les effets de la magnétostriction spontanée sur la susceptibilité perpendiculaire de $\mathrm{MnO}$ et nous avons pu les relier à d'autres observations telles que le retard manifesté dans l'apparition de la distorsion rhomboédrique et l'hystérésis de la susceptibilité en fonction de la température.

En raison des limites imposées par l'appareillage l'étude de MnS n'a pas pu être aussi concluante, et des mesures dans des champs plus intenses, pulsés ou statiques, sont très souhaitables. Dans ce composé comme dans $\mathrm{MnO}$, les mouvements des domaines ne peuvent être négligés.

\section{Bibliographie}

[1] Lindsay (R.) et Michelson (F. H.), Bull. Amer. Phys. Soc., 1966, 11, 108, et communication personnelle.

[2] Lindsay (R.) et Banewicz (J. J.)., Phys. Rev., 1958, $110,634$.

[3] Lines (M. E.) et Jones (E. D.), Phys. Rev., 1965, $139 \mathrm{~A}, 1313$.

[4] Lines (M. E.) et Jones (E. D.), Phys. Rev., 1966, 141, 525.

[5] Shul (C. G.), Strauser (W. A.) et Wollan (E. O.), Phys. Rev., 1951, 83, 333.

[6] Kaplan (J. I.), J. Chem. Phys., 1954, 22, 1709.

[7] Roth (W. L.), Phys. Rev., 1958, $110,1333$.

[8] Bizette (H.), Squire (C. F.) et Tsai (B.), C. R. Acad. Sci. Paris, 1938, 207, 449.

[9] Néel (L.), C. R. Acad. Sci. Paris, 1936, 203, 304.

[10] Bartholin (H.), Bloch (D.) et Georges (R.), $C . R$. Acad. Sci. Paris, 1967, 264 B, 360.

[11] Bloch (D.), Charbit (P.) et Georges (R.), $C . R$. Acad. Sci. Paris, 1968, 266 B, 430.

[12] Georges (R.), C. R. Acad.Sci. Paris, 1969, 268 B, 16.

[13] Boissier (R.), Brichant (F.), Goyer (J.), Fournier (J.), Riceue (R.), Feron (J. L.), Pauthenet (R.) et Picoche (J. C.), « Les Champs Magnétiques Intenses, leur Production et leurs Applications "), C. N. R. S. éditeur, 1967.

[14] Selwood (P. W.), Magnetochemistry, Interscience Publishers, Inc., New York, 1956.

[15] Roth (W. L.), J. Appl. Phys., 1960, 31, 2000.

[16] Slack (G. A.), J. Appl. Phys., 1960. 31, 1571.

[17] Saito (S.), J. Phys. Soc. Japan, 1962, 17, 1287.

[18] KondoH (H.), J. Phys. Soc. Japan., 1962, 17, 1316.

[19] Georges (R.), Thèse, Grenoble, 1969.

[20] Bloch (D.), Feron (J. L.), Georges (R.) et Jacobs (I. S.), J. Appl. Phys., 1967, 38, 1474.

[21] Rodbell (D. S.), Jacobs (I. S.), OWen (J.) et Harris (E. A.), Phys. Rev. Letters, 1963, 11, 10.

[22] Keffer (F.) et O'Sullivan (W.), Phys. Rev., 1957, $108,637$.

[23] Rodbell (D. S.) et Owen (J.), J. Appl. Phys., 1964, 35,1002 , et communication personnelle.

[24] Georges (R.), à paraître.

[25] Kubo (R.), Phys. Rev., 1952, 87, 568.

[26] Kondoh (H.) et Takeda (T.), J. Phys. Soc. Japan, 1964, 19, 2041.

[27] MollaRd (P.), communication personnelle. 